ARTICLE

DOI: $10.1038 / s 41467-017-00416-8$

\title{
Modifying electron transfer between photoredox and organocatalytic units via framework interpenetration for $\beta$-carbonyl functionalization
}

\author{
Zhengqiang Xia ${ }^{1}$, Cheng $\mathrm{He}^{1}$, Xiaoge Wang ${ }^{1} \&$ Chunying Duan ${ }^{1,2,3}$
}

Modifying electron transfer pathways is essential to controlling the regioselectivity of heterogeneous photochemical transformations relevant to saturated carbonyls, due to fixed catalytic sites. Here we show that the interpenetration of metal-organic frameworks that contain both photoredox and asymmetric catalytic units can adjust the separations and electron transfer process between them. The enforced close proximity between two active sites via framework interpenetration accelerates the electron transfer between the oxidized photosensitizer and enamine intermediate, enabling the generation of $5 \pi \mathrm{e}^{-} \beta$-enaminyl radicals before the intermediates couple with other active species, achieving $\beta$-functionalized carbonyl products. The enriched benzoate and iminium groups in the catalysts provide a suitable Lewis-acid/base environment to stabilize the active radicals, allowing the protocol described to advance the $\beta$-functionalization of saturated cyclic ketones with aryl ketones to deliver $\gamma$-hydroxyketone motifs. The homochiral environment of the pores within the recyclable frameworks provides additional spatial constraints to enhance the regioselectivity and enantioselectivity.

\footnotetext{
${ }^{1}$ State Key Laboratory of Fine Chemicals, Dalian University of Technology, Dalian 116024, China. ${ }^{2}$ College of Zhang Dayu, Dalian University of Technology, Dalian 116024, China. ${ }^{3}$ Collaborative Innovation Center of Chemical Science and Engineering, Tianjin 300071, China. Correspondence and requests for materials should be addressed to C.D. (email: cyduan@dlut.edu.cn)
} 
C atalytic synthesis methods that work under ambient atmosphere with benign reaction environments and clean energy have been a major goal in synthetic chemistry ${ }^{1}$. Of the essential structural motifs that are frequently found in pharmaceutical, material, agrochemical, and fine chemicals, the carbonyls play a pivotal role as powerful building blocks in broad areas of synthetic organic chemistry ${ }^{2-4}$. The functionalization of carbonyls, one of the most fundamental transformation in organic synthesis, has evolved to a range of widely used organic reactions and synthetic protocols ${ }^{5}$. While direct $\alpha$-carbonyl substitution is flourishing because the $\alpha$-methylene position of the carbonyl group can be readily functionalized with diverse nucleophiles and electrophiles via enolate or enamine chemistry $^{6-8}$, the direct $\beta$-activation of saturated carbonyls has proven to be a more cumbersome and challenging task owing to the typically unreactive $\beta-\mathrm{C}\left(\mathrm{sp}^{3}\right)-\mathrm{H}$ bonds and competitive $\alpha$ substitution or other reactions ${ }^{9-11}$. Most primary $\beta$-carbonyl substitution is still achieved indirectly by the nucleophilic addition of $\alpha, \beta$-unsaturated carbonyls ${ }^{12,13}$, despite a few examples of direct $\beta$-activation having been reported by enamine oxidation or palladium catalysis ${ }^{14,15}$

On the other hand, light-promoted photocatalysis, a mild and green chemical synthesis approach, furnishes powerful molecular activation modes to enable bond constructions that are elusive or impossible via established protocols ${ }^{16,17}$, undoubtedly granting a new opportunity for the direct $\beta$-position bonding of saturated carbonyls. Recent investigation has also suggested that the combination of photoredox catalysis with enamine organocatalysis is a promising strategy for the $\beta$-functionalization of saturated carbonyls through a $5 \pi \mathrm{e}^{-}$carbonyl activation mode $\mathrm{e}^{18}$. It is postulated that the clean energy activation mode applies to a wide range of $\beta$-carbonyl functionalization transformations and is also compliable with asymmetric catalysis; meanwhile, the long-life and high-energy radical in such homogeneous system requires the co-existence of several types of catalysts and adducts to enhance its stability and ensure effective coupling reactions.
However, in heterogeneous systems, owing the fixed catalytic sites, the modification and optimization of the rapid electron transfer between the two catalytic cycles for regioselectivity control should be given important consideration during the design of the catalysts. In this case, the major challenge goes beyond the incorporation of suitable photoredox centers and organocatalytic centers within the catalytic systems and includes the precise regulation of the electron transfer pathway between the active sites. New strategies wherein the location of all the catalytic requisites can be modified in an easy-to-control model with adjustable electron states and interactional patterns appear imminent to achieve photocatalytic transformation.

Metal-organic frameworks (MOFs) are fascinating hybrid solids with infinitely ordered networks consisting of organic bridging ligands and inorganic nodes ${ }^{19}, 20$. The tenability and flexibility of the polymers granted by the diversity of their building blocks allow the incorporation of photoactive organic dyes and the necessary adducts into a single framework, which represents a new approach to heterogenizing several photocatalytic conversions ${ }^{21}$. The regular distribution of catalytic sites within the confined micro-environment benefits the fixation and stabilization of the active radicals formed under irradiation to overcome restrictions of homogeneous processes ${ }^{22}$. The longrange ordered structure of a MOF-based crystalline material contains precisely located catalytic centers, which provides an excellent platform to study photon capture and electron transport and delivery ${ }^{23,24}$. As the integration of both photocatalysis and asymmetric organocatalysis into a single MOF has been considered a promising approach to the $\alpha$-functionalization of saturated carbonyls ${ }^{25}$, we believe the modification of electron transfer between the two active sites within the frameworks via framework interpenetration is a potential strategy for the $\beta$ functionalization of saturated ketones and aldehydes.

Here we show that the interpenetration of the polymers allowed the appropriate adjustment of the separation and interactions between the photoredox and organocatalytic sites. With
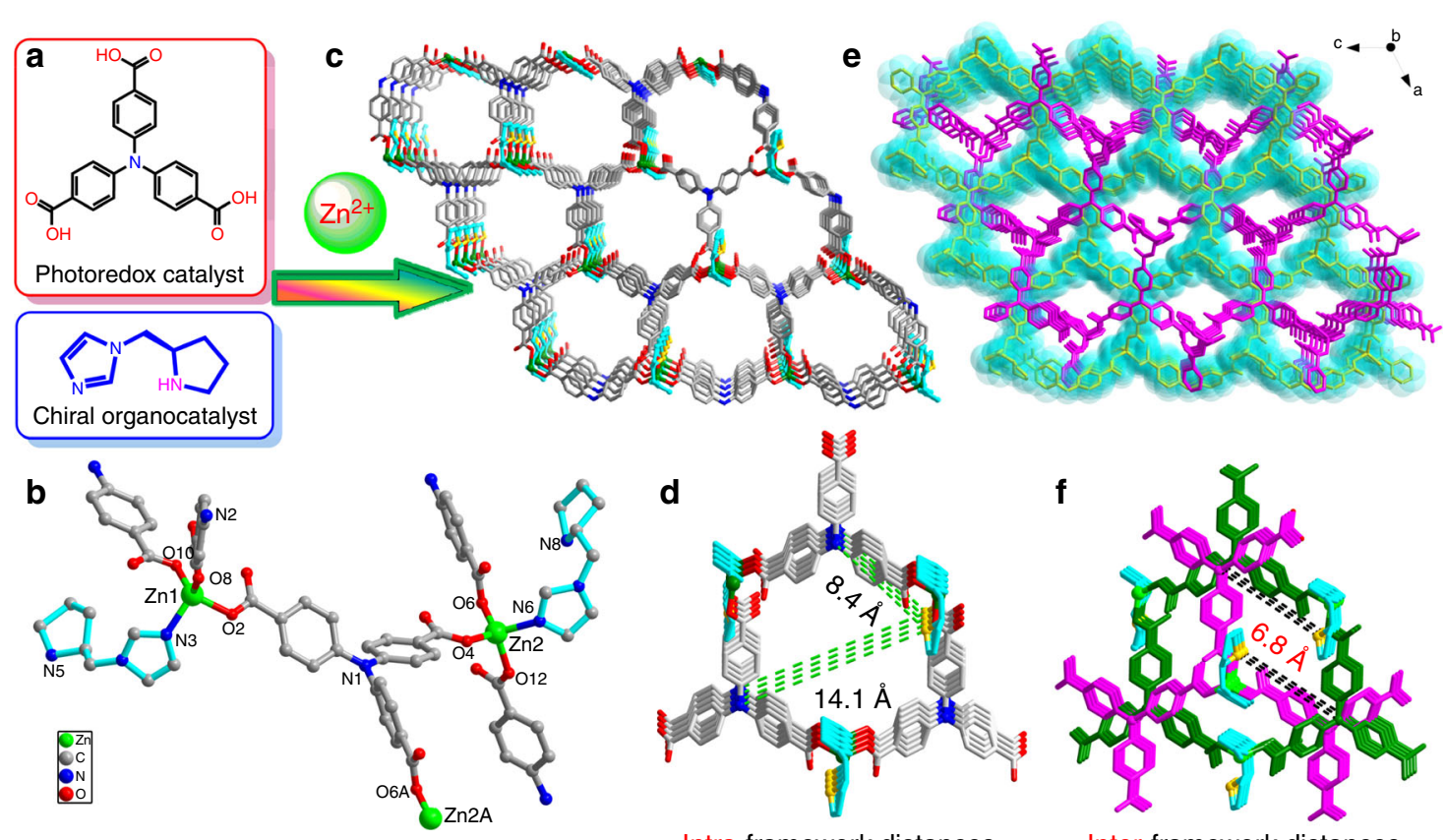

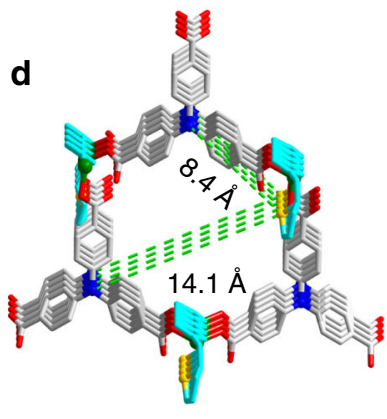

Intra-framework distances

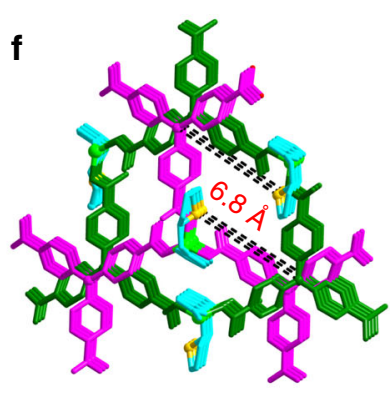

Inter-framework distances

Fig. 1 The assembly and crystal structure of InP-1. The components (a) and coordination environments of the Zn(II) ions and NTB ${ }^{\mathbf{3}^{-}}$ligand (b) in InP-1; Symmetry code: $\mathrm{A}, x+1 / 2, y-1 / 2, z$. 3D structures of the isolated frameworks viewed along the $b$-axis, before $\mathbf{c}$ and after $\mathbf{e}$ the twofold interpenetration. The intra-(d) and inter-(f) framework separations between the catalytic sites. Atoms in the pyrrolidine rings of L-PYI are marked in yellow and turquoise. $\mathrm{H}$-atoms and solvent molecules are omitted for clarity 
the closer interframework distances, the electron transfer between the two active sites is accelerated, such that the special $5 \pi \mathrm{e}^{-} \beta$ enaminyl radical is more likely to be quickly generated from the enamine intermediate to achieve $\beta$-functionalized carbonyl products before the enamine intermediate is coupled with other active species. As a heterogeneous photocatalysis for the direct $\beta$ functionalization of saturated carbonyls, the ability to recover and reuse the photocatalysts makes our system interesting in terms of the practical applications of the photochemical transformation. Additionally, the enriched benzoate and imine groups in the polymers create a suitable Lewis acidic/basic environment to replace the complex catalytic adducts in a homogeneous system to further stabilize long-life and high-energy radicals, which makes the catalytic system simpler, milder and more environmentally friendly. The matched redox potentials of the interpenetrated polymers promoting this catalytic protocol are further advanced to allow the $\beta$-functionalization of the other hard-toreduce saturated carbonyls.

\section{Results}

Synthesis and characterization of the interpenetrated MOF. Solvothermal reaction of $L$-pyrrolidin-2-ylimidazole $(L \text {-PYI })^{26}$, $4,4^{\prime}, 4^{\prime \prime}$-nitrilotrisbenzoicacid $\left(\mathbf{H}_{3} \mathbf{N T B}\right)$, and $\mathrm{Zn}\left(\mathrm{NO}_{3}\right)_{2} \cdot 6 \mathrm{H}_{2} \mathrm{O}$ afforded a hybrid crystalline solid, InP-1 (Fig. 1a, Supplementary Data 1). Elemental analysis and powder X-ray diffraction demonstrated the potential phase purity of the bulk sample (Supplementary Fig. 1). Single-crystal X-ray diffraction revealed the formation of the twofold interpenetrated coordination polymer and the crystallization into the chiral space group $C_{2}$. Within each of the identically isolated chiral framework, the $\mathrm{Zn}$ (II) ion was coordinated in a chiral tetrahedral geometry with three carboxylate oxygen atoms from three different $\mathbf{N T B}^{\mathbf{3}-}$ ligands and one nitrogen atom from the PYI coligand. The propeller-like NTB $^{3-}$ ligand rotated the arms with special angles to meet the requirements of the $\mathrm{Zn}$ (II)-tetrahedron geometries (Fig. 1b) and linked three zinc ions through the carboxylate groups to generate a 3D framework containing 1D chiral channels with a cross section of $16.7 \times 17.0 \AA^{2}$ (Fig. 1c). Two symmetric related frameworks were interpenetrated to form the crystalline solid, with the opening of the channels being reduced to $14.3 \times 9.4 \AA^{2}$ (Fig. 1e, Supplementary Figs. 9 and 10). Chiral PYI catalytic sites were regularly distributed on the inner surfaces of the channels with the exposed active amine groups $(\mathrm{N}-\mathrm{H}$ pyrrolidine) working as accessible catalytic sites to active aldehydes or ketones.

Most importantly, the interpenetration of the two frameworks enforced the close proximity between the photoredox sites $\left(\mathbf{N T B}^{\mathbf{3}}\right)$ and the asymmetric organocatalytic sites (PYI), enabling the shortest intraframework $\mathrm{N} \cdots \mathrm{N}$ separation of $8.4 \AA$ between the pyrrolidine and triphenylamine $\mathrm{N}$ atoms in each non-interpenetrating framework aforementioned shorten to $6.8 \AA$ in the interpenetrated InP-1 (Fig. 1d, f). The formed structural motif will benefit the acceleration of the electron transfer between the enamine intermediate and the photosensitizer. This acceleration promotes the generation of the $5 \pi \mathrm{e}^{-} \beta$-enaminyl radical, a key step for the achievement of the $\beta$-activation of carbonyl, being
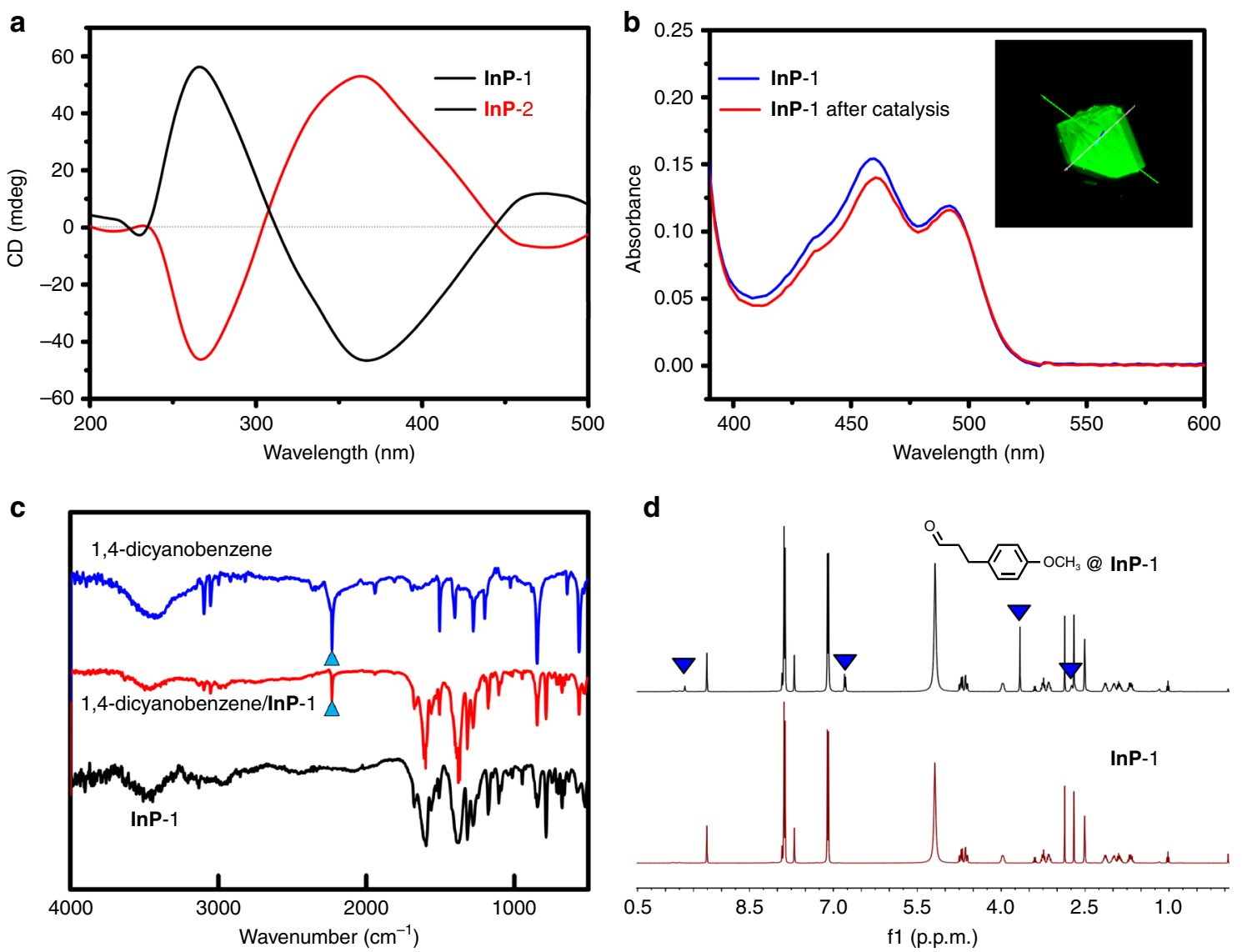

Fig. 2 The chirality and porosity characterization of InP-1. a CD spectra of bulk crystalline solids of InP-1 and InP-2 showing the opposite Cotton effects of the two polymers. b UV-vis spectra of $2^{\prime}, 7^{\prime}$-dichloro fluorescein dye released from InP-1 and InP-1 after catalysis. Inset: the 3D reconstruction of InP-1 soaked with 2', $7^{\prime}$-dichlorofluorescein dye. c IR spectra of 1,4-dicyanobenzene, InP-1, and InP-1 with absorbed 1,4-dicyanobenzene. The light blue triangles show the characteristic $\nu^{\text {st }} \mathrm{C} \equiv \mathrm{N}$ signals of 1,4-dicyanobenzene. $\mathbf{d}^{1} \mathrm{H}$ NMR spectra of InP-1 and InP-1 with adsorbed 3-(4-methoxyphenyl) propionaldehyde dissolved in DMSO- $d_{6} / \mathrm{DCl}$. Peaks marked with blue triangles represent the signals of 3-(4-methoxyphenyl) propionaldehyde 
more rapid than the direct coupling reaction of the enamine intermediate with other active species. Additionally, the free migration of protons around the uncoordinated nitrogen or oxygen atoms of the ligands and the chiral organocatalysts allows the enriched Lewis acid and Lewis base to possibly act as catalytic adducts to stabilize high-energy radicals. This structural motif grants a possibility of replacing the complex catalytic adducts of the homogeneous system ${ }^{18}$, making the reaction conditions simpler, cleaner and more environmentally friendly, and allowing separation, and recovery in potential practical applications.

The chirality of the interpenetrated frameworks was identified by the chiral space group it crystallized into and confirmed by the circular dichroism (CD) spectrum, with a positive Cotton effect centered at $\sim 263 \mathrm{~nm}$ and a negative Cotton effect centered at $\sim 366 \mathrm{~nm}$ (Fig. 2a and Supplementary Table 1). The porosity of the polymer was determined by the PLATON software ${ }^{27}$ with $18.8 \%$ of the whole crystal volume calculated. To evaluate the accessibility and stability of the channels to the bulky organic substrates in the solution environment, a dye-uptake assay, an increasingly common method was performed before the MOFbased catalyst was used in catalytic investigations ${ }^{28,} 29$. In the general procedure, the as-synthesized crystalline solid InP-1 was first soaked overnight in a methanol solution containing $2^{\prime}, 7^{\prime}$ dichlorofluorescein. The resulting crystalline solid was filtered and washed with methanol several times to remove the potential dye molecules adsorbed on the external surfaces of the sample. The dye-absorbed InP-1 was then dissociated by concentrated hydrochloric acid, and the resultant clear solution was diluted to $10 \mathrm{~mL}$ and adjusted to a $\mathrm{pH}$ of 1.5 . The quantum dye-uptake was determined as $12.5 \%$ of the solid weight by ultraviolet-visible (UV-vis) spectroscopy (Supplementary Fig. 6). Confocal laser scanning microscopy of the dye-adsorbed crystal showed the successful uptake of the dye molecules within the channels of the polymer (Fig. 2 b and Supplementary Fig. 7$)^{30,31}$, which revealed the good stability of the well-maintained pores in solution and the possibility of the substrates diffusing inside the channels.

To further confirm the capability of InP-1 to transport substrate molecules through the open channels, the InP-1 was immersed in solutions of $\mathrm{CH}_{2} \mathrm{Cl}_{2}$ containing the possible substrates 1,4-dicyanobenzene or 3-(4-methoxyphenyl)propionaldehyde. Infrared (IR) spectrum of InP-1 with adsorbed 1,4dicyanobenzene showed the characteristic signals of $\nu^{s t} \mathrm{C} \equiv \mathrm{N}$ at $2231 \mathrm{~cm}^{-1}$ (Fig. 2c), and the ${ }^{1} \mathrm{H}$ NMR of InP-1 with adsorbed substrates revealed that InP-1 could adsorb $\sim 1.3$ equiv of 1,4dicyanobenzene or 1 equiv of 3-(4-methoxyphenyl)propionaldehyde per triphenylamine moiety (Fig. 2d and Supplementary Fig. 20). All these results demonstrated that the pores of the MOFs are accessible for smooth substrate diffusion and suggested that InP-1 was suitable for heterogeneous catalysis. Furthermore, because the InP-1 was evolved from two of the same noninterpenetrating frameworks via interpenetration, this transformation provides a chance of adjusting the pore environment and at the same time enhancing the density of triphenylamine groups around the pores.

Photocatalytic $\boldsymbol{\beta}$-functionalization of saturated aldehydes. The $\beta$-arylation reaction was initially examined by employing 1,4 dicyanobenzene and propionaldehyde as the coupling partners, along with a 20 watt fluorescent lamp as the light source. A $5 \mathrm{~mol}$ $\%$ loading of InP-1 gives an $87 \%$ conversion to the $\beta$-functionalization under irradiation without the formation of any $\alpha$ functionalization adduct (entry 1, Table 1). Compared with the complex reaction conditions (multiple additives: base, water, acid, photosensitizer, catalyst, and 1,3-dimethyltetrahydropyrimidin-2 $(1 H)$-one (DMPU) solvent) in the recently reported homogeneous $\beta$-functionalization of carbonyls ${ }^{18}$, our catalytic system afforded efficient and selective transformation in the presence of only the organic base 1,4-diazabicyclo[2.2.2] octane (DABCO) and the designed MOF-based catalysts in N,N-dimethylformamide (DMF) solution, which made our reaction conditions simpler, milder, and more manageable. Moreover, the heterogenization of the organic photoredox catalyst $\mathbf{H}_{3}$ NTB into the frameworks replaced the noble-metal $\operatorname{Ir}(\text { ppy })_{3}$ in the homogeneous systems, which not only prevented heavy metal pollution on final reaction products but also saved the costs of synthesis and post-processing in mass production, rendering our system less costly and more environmentally friendly.

The InP-1 solids were easily isolated from the reaction suspension by simple filtration. The removal of InP-1 by filtration after $12 \mathrm{~h}$ of irradiation shut down the reaction directly, with the filtrate affording only $4 \%$ additional conversion over another $36 \mathrm{~h}$ of irradiation (Fig. 3a). Washing the filtered residues with DMF enabled their direct reuse in a new round of reactions. After three rounds of reuse, InP-1 solids exhibited a moderate loss of reactivity ( $87 \sim 82 \%$ conversion; Fig. $3 \mathrm{~b}$ and Supplementary Table 5). The PXRD pattern of the InP-1 filtered from the reaction mixture matched well with the simulated one based on the single-crystal simulation, suggested the maintenance of the MOF framework (Supplementary Fig. 1). Additionally, further confirming the maintenance of the pores of the catalysts, dyeuptake studies for the recycled InP-1 exhibited an $11.7 \%$ dyeuptake corresponding to the framework weight. The comparable adsorption ability before and after the catalytic reaction confirmed the chemical stability of the well-preserved pores (Fig. 2b). The results suggested the successful execution of our structural design and demonstrated that InP-1 was a heterogeneous catalyst with advantages of easy separation and recovery $^{32}$ that utilizes mild and more environmentally friendly reaction conditions and has potential practical applications.

Control experiments showed that no transformation occurred in the presence of only the $5 \mathrm{~mol} \% \mathbf{H}_{3} \mathbf{N T B}$, the $5 \mathrm{~mol} \%$ PYI or the 5 equiv of DABCO base. Almost negligible conversion was observed when the reaction was performed in the dark (entries 1-5, Supplementary Table 4). To directly compare the MOFcatalyzed reaction with the standard homogeneous conditions ${ }^{18}$, the same adducts and solvent (DABCO, HOAc, water, and DMPU) in equivalent amounts were added to catalyze the transformation with free catalysts, but no $\beta$-arylation product was detected. This result suggested that the interpenetrated MOF catalysts played an indispensable role in our heterogeneous system (entry 6, Supplementary Table 4). Considering the catalytic functions of $\mathbf{H}_{3} \mathrm{NTB}$ and PYI, $5 \mathrm{~mol} \% \mathrm{H}_{3} \mathrm{NTB}$ and PYI were loaded as the homogeneous catalysts to catalyze the reaction under optimal conditions. A low conversion of $11 \%$ was observed because the arms of $\mathbf{H}_{3}$ NTB rotated quickly in the solvent and gave very poor light absorption (entry 8, Supplementary Table 4$)^{33-35}$. Even when adding extra zinc salts to coordinate with the $\mathbf{H}_{3}$ NTB and thereby hinder the rotation, only a small increase to $26 \%$ conversion could be obtained.

A further comparison of the catalytic efficiency was based on an interpenetrated achiral coordinated polymer (InP-3) with almost the same structure, except the chiral organocatalytic site was substituted by a pyridine moiety (Fig. 3c, Supplementary Data 3 and Supplementary Fig. 11). As expected, the loading of 5 mol\% InP-3 under the same reaction conditions as InP-1 yielded negligible conversion. The two structurally similar compounds have almost the same ground redox potential $\left(E_{1 / 2} \approx 0.83 \mathrm{~V}\right.$ vs. SCE) in the solid state and quite similar absorption and emission spectra, and the excited state redox potentials of $-2.16 \mathrm{~V}$ and $-1.97 \mathrm{~V}$ of InP-1 and InP-3, respectively (Supplementary Figs. 4 and 5$)^{36}$, are both more negative than those of organomatallic Ir 
Table 1 Conversion and enantiomeric excess (ee) of the photocatalytic $\beta$-arylation of saturated aldehydes with InP-1 and InP-2 as bifunctional heterogeneous catalysts ${ }^{a}$

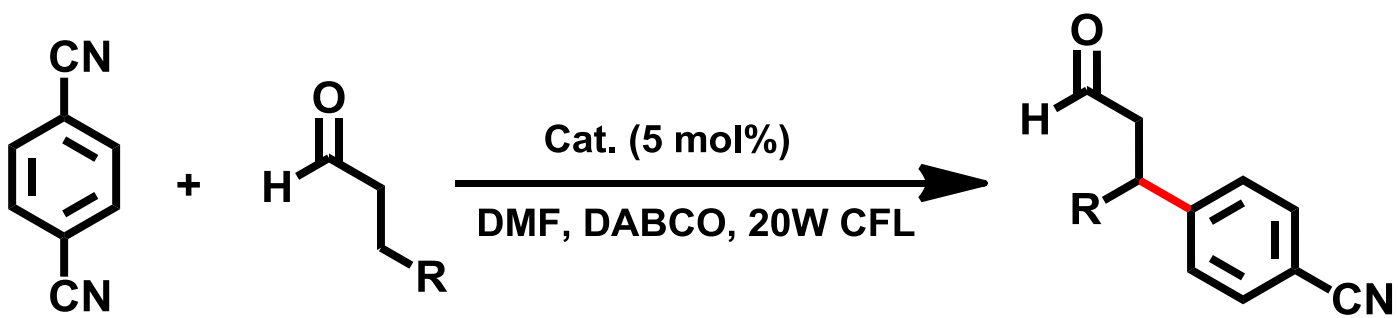

Entry $\quad$ Substrate $^{\mathrm{b}} \quad$ Molecular size ${ }^{\mathrm{b}} \quad$ Conversion $/ \%^{\mathrm{c}} \quad$ ee $/ \%^{\mathrm{d}}$

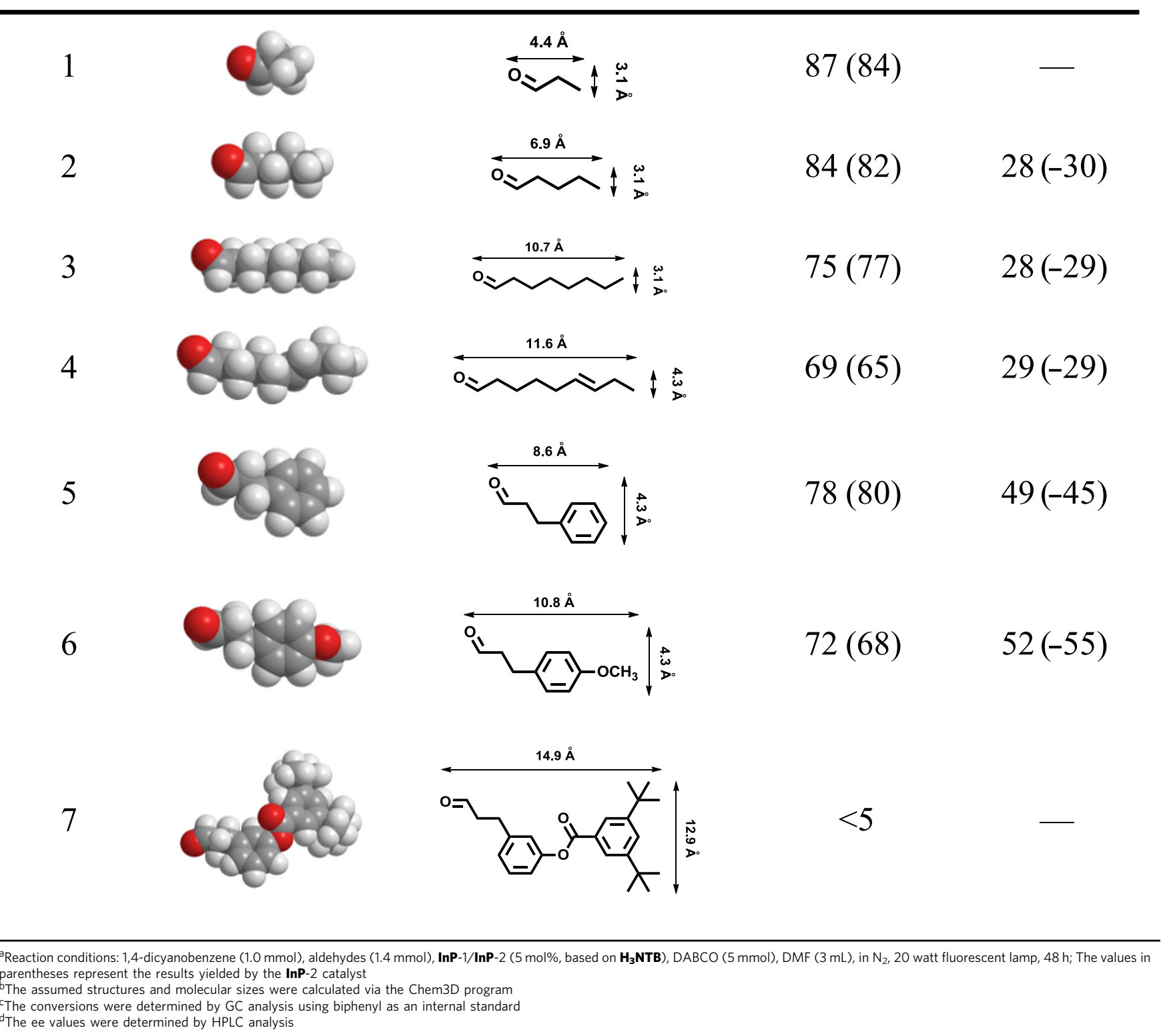

$(\text { ppy })_{3}\left(E_{1 / 2}{ }^{\text {ox }}=-1.73 \mathrm{~V}\right)^{37,38}$ and the substrate 1,4-dicyanobenzene $\left(E_{1 / 2} \text { red }=-1.61 \mathrm{~V}\right)^{39}$. The difference in the catalytic efficiency is attributed to the absence of synergistically organocatalytic groups that used to activate the substrate. This postulation was confirmed by the conversion of the aforementioned catalytic system increasing to $58 \%$ upon the excess addition of the organocatalytic $L$-PYI to the reaction mixture.

From a mechanistic point of view, in our heterogeneous system, the interpenetrated coordinated polymer InP-1 adsorbed 

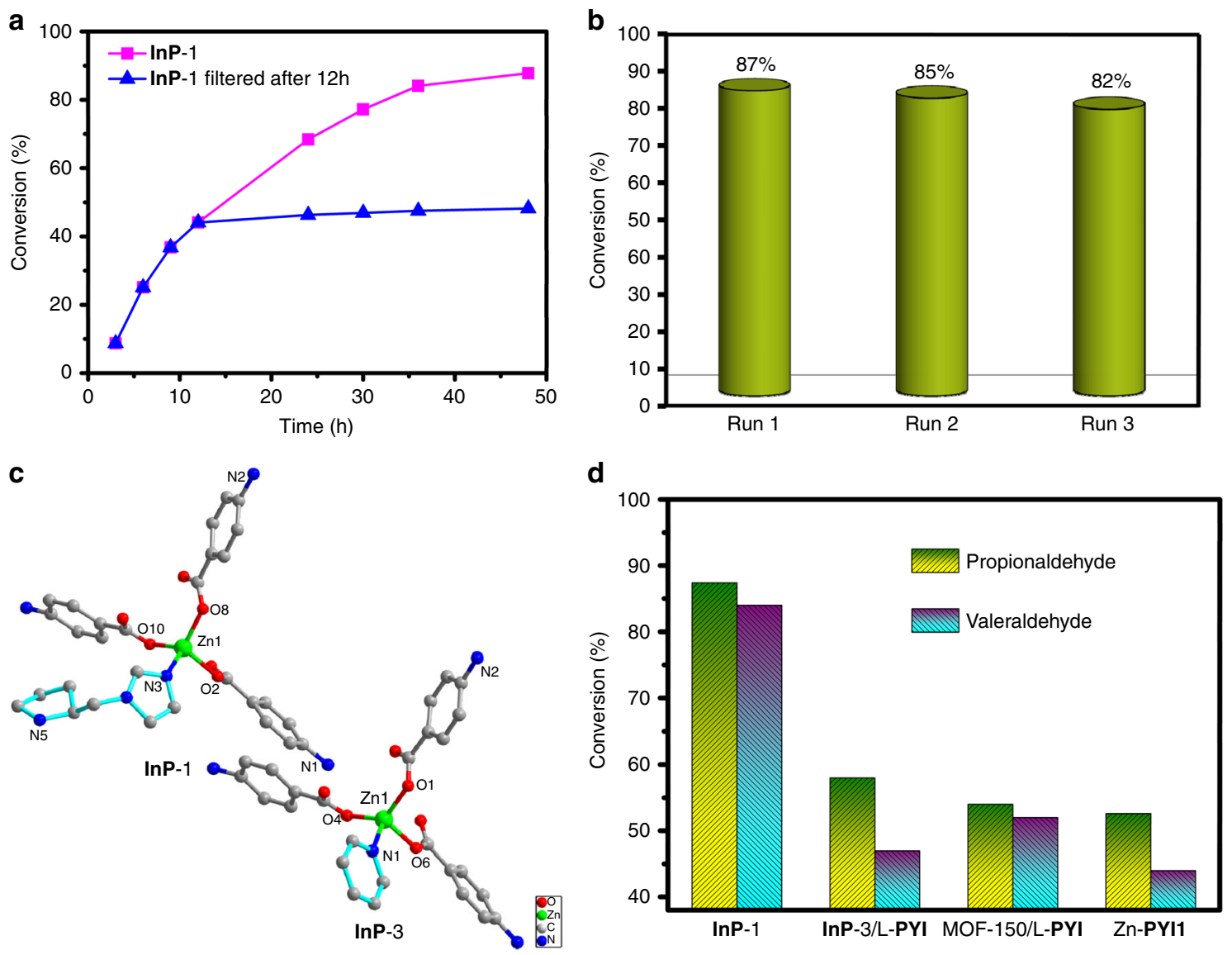

Fig. 3 The photocatalysis results and control experiments. a Catalytic traces of the $\beta$-arylation of propionaldehyde performed by InP-1 and InP-1 filtered after $12 \mathrm{~h}$ under the optimal conditions. b Recycling catalytic experiments of the $\beta$-arylation of propionaldehyde catalyzed by InP-1 under the optimum conditions. c The coordination environments of $\mathbf{Z n}$ (II) ions in InP-1 and InP-3. d Conversion levels of the $\beta$-arylation reaction between 1,4-dicyanobenzene and saturated aldehydes using different catalysts ( $5 \mathrm{~mol} \%$ )

1,4-dicyanobenzene within its channels, and this substrate quenched the excited state of the heterogeneous catalyst to afford the radical anion and oxidized InP- $1^{+}$. The aldehyde substrates readily diffuse inside the channels and are activated by the chiral amine catalyst PYI moiety to potentially form the normal chiral enamine intermediate. The close proximity between the photoredox catalytic site and the organocatalytic site increased the opportunity for the enamine intermediate to be quickly oxidized by InP $-1^{+}$through interframework electron transfer to generate the corresponding enaminyl radical cation, rather than direct coupling with the arene radical anion or other species that created bonds at the $\alpha$-carbonyl position. The high-energy enaminyl radical cations were favorable to increase the acidity of the allylic $\mathrm{C}-\mathrm{H}$ bonds and further promote the corresponding deprotonation at the $\beta$-position of carbonyl, eventually completing the critical $5 \pi \mathrm{e}^{-}$activation mode. The newly formed $5 \pi \mathrm{e}^{-} \beta$-enaminyl radical quickly coupled with the 1,4-dicyanobenzene radical anion to yield the cyclohexadienyl anion, which further underwent hydrolysis to give the $\beta$-arylation product and return the PYI (Fig. 4 and Supplementary Fig. 15). This prospective is confirmed by the luminescent quenching of the interpenetrated polymer upon the addition of $50 \mu \mathrm{M}$ 1,4-dicyanobenzene substrate, with Stern-Volmer constants of the constructed polymers InP-1 and InP-3 of $3.56 \times 10^{3}$ and $3.83 \times 10^{3} \mathrm{M}^{-1}$, respectively. The corresponding fluorescence lifetime decreased from 4.62 to $3.25 \mathrm{~ns}$, which revealed a classical photo-induced electron transfer process from the excited state of the catalyst to the substrate and a fast charge transfer rate (Supplementary
Figs. 21-24) $)^{40}$. The IR spectrum of InP-1 immersed in a DMF solution of propionaldehyde revealed a $\mathrm{C}=\mathrm{O}$ stretching vibration at $1724 \mathrm{~cm}^{-1}$. The redshift from $1736 \mathrm{~cm}^{-1}$ (free propionaldehyde) suggested the adsorption and activation of propionaldehyde in the pores of the polymer (Supplementary Fig. 16). These affinities implied the probability of the formation of the key enamine intermediate. Additionally, the fact that the relevant shift was not observed in IR spectrum of InP-3-adsorbed propionaldehyde confirmed the inactivity of InP-3 under the catalytic processes (Supplementary Fig. 25).

The unique crystalline characteristic of the MOFs yields the opportunity to learn their complex internal structure by singlecrystal X-ray diffraction technique. In addition, the precise determination of the crystal structure of the frameworkencapsulated substrate molecules provides more chemical information about the host-guest interaction patterns, paths, and sites distribution, which allows us to better understand the mysterious activation and formation processes of the catalytic intermediate and has become a promising approach for catalytic studies ${ }^{41,42}$. Thus, the InP-1 crystals were soaked in a solution containing substrates for $12 \mathrm{~h}$, and fortunately the quality of the propionaldehyde-impregnated InP-1 crystals (denoted InP-4) was suitable for X-ray crystallographic analysis (Supplementary Data 4 and Supplementary Figs. 12 and 18). The structural analysis of InP-4 revealed that the space group and unit cell sizes were almost the same as those of the original crystal. As shown in Fig. $5 \mathrm{a}$, the propionaldehyde substrates were perpendicularly embedded in the channels, and the asymmetric unit of InP-4 


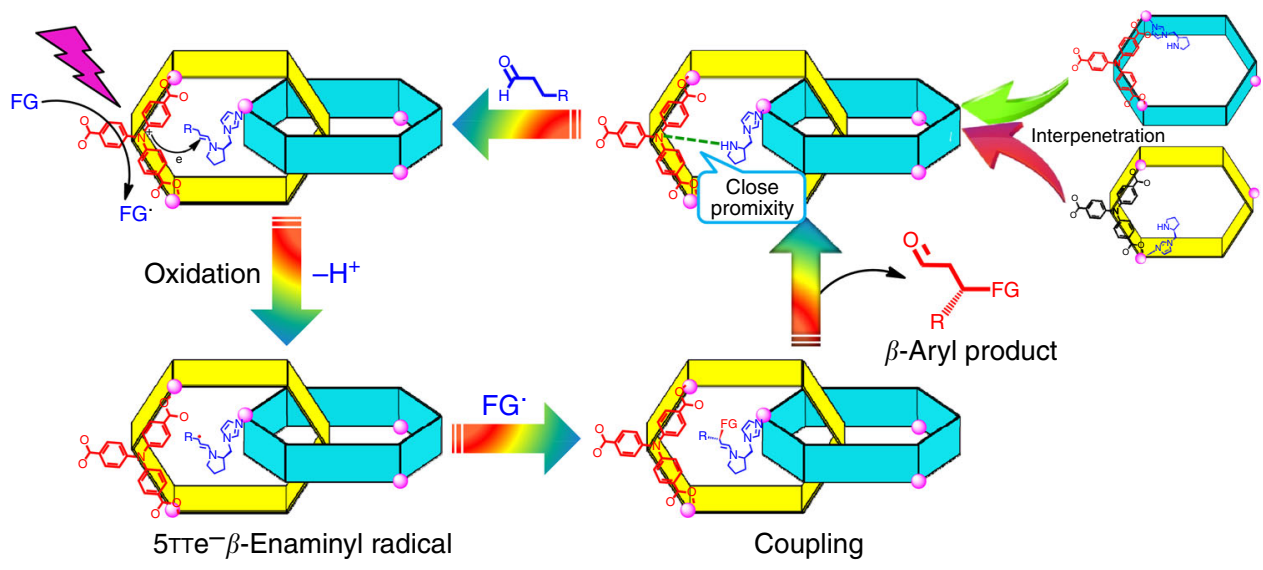

Fig. 4 Proposed mechanisms for interpenetrated MOFs in photocatalytic $\beta$-carbonyl activation. Representation of the electron transfer pathway relevant to the photoredox and organocatalytic units via framework interpenetration for the $\beta$-functionalization of saturated aldehydes. $F G$ functional group; $R$ generic organic substituent

included 1.5 propionaldehyde molecules. Each substrate molecule was disordered and divided into two parts because of molecular thermal movement. One part of the carbonyl groups was located close to the PYI moiety, and the shortest distance between the pyrrolidine nitrogen and the carbonyl oxygen atoms of propionaldehyde was $2.97 \AA$. Such a close distance demonstrated a substrate-framework $\mathrm{N}-\mathrm{H}$...O hydrogen bond, which may provide an additional driving force to enforce the substrate approaching the active amine catalytic sites closely and facilitating the enamine activation. Several strong $\mathrm{O}-\mathrm{H} . . . \mathrm{O}$ hydrogen bond interactions $(2.68$ and $2.35 \AA$ ) were also observed between the other part of the carbonyl groups and the free solvent molecules (O15 and O16), which was postulated to be the driving force for the capture and stable location of the substrate within the channels of the catalyst framework.

When valeraldehyde and other more complex aldehydes were used as substrates (entries 2-6, Table 1), conversions between 69 and $84 \%$ were achieved in the InP-1 reaction systems, with the enantio excess reaching 52\% (Supplementary Figs. 28-39 and 55-59). The insertion of the aromatic aldehyde radical coupling partners in the pores caused stronger aromatic stacking interactions and a better enantioselectivity than that of the aliphatic aldehyde product. It should be noted that these produced enantioselectivities were not found in the reported homogeneous work ${ }^{18}$ or our control experiment (entry 1, Supplementary Table 7). These results suggested that the interpenetrated frameworks with confined chiral environments may enhance the enantioselectivity of the $\beta$-functionalization products. Interestingly, the selectivity is comparable to the best enantio excess achieved in homogeneous $\beta$-arylation reactions between 1,4-dicyanobenzene and cyclohexanone, which use chemical cocktail reaction mixtures and a larger loading (20\%) of a quite complex chiral organoamine as the chiral director ${ }^{18}$. The simple reaction mixture in our system not only optimized the electron transfer and activation pathways to achieve efficient $\beta$ arylation transformation but also provided additional spatial constraints to enhance the enantioselectivity without introducing any additional chiral sources, which demonstrates such interpenetrated MOFs to be promising catalysts for the asymmetric photocatalytic transformation. When $L$-PYI was replaced by $D$ pyrrolidin-2-yl-imidazole ( $D$-PYI), the other enantiomorph, InP2 (Supplementary Data 2 and Supplementary Figs. 2 and 8), was isolated with the same cell dimensions as and a mirror-image structure to InP-1, exhibiting similar catalytic activity but granting products with opposite chirality under the optimum conditions (Supplementary Table 3). Interestingly, similar substrate activation was also found in InP-2 systems. The valeraldehyde-impregnated InP-2 crystals (denoted InP-5) with good quality were fortunately obtained, and the single-crystal Xray diffraction analysis suggested the maintenance of space group and main framework (Supplementary Data 5 and Supplementary Figs. 12 and 19). As depicted in Fig. 5b, the asymmetric unit of InP- 5 contained one valeraldehyde molecule, and each substrate was perpendicularly inserted in the center of the channel. Such a special spatial occupation with the minimum steric hindrance in the channel, suggested the smooth diffusion of the substrates. The exposed active amine groups of PYI (N5-H5) around the inner walls of the channels provided an affinity to the carboxyl oxygen atoms of aldehyde (O17) with the $\mathrm{N}-\mathrm{H} . . . \mathrm{O}$ hydrogen bond $(2.85$ $\AA$ ) interactions, which could be the potential activation forms and were extremely beneficial to the formation of enamine intermediate. Such carbonyl activation was further supported by the redshift of $\mathrm{C}=\mathrm{O}$ stretching vibration from $1736 \mathrm{~cm}^{-1}$ (free valeraldehyde) to $1710 \mathrm{~cm}^{-P}$ between the IR spectra of InP-5 and valeraldehyde (Supplementary Fig. 17).

When the bulky aromatic aldehyde (E)-3-(3-oxoprop-1-enyl) phenyl 3,5-di-tert-butylbenzoate was used as a substrate ${ }^{43}$, a $<5 \%$ conversion under the same reaction condition was observed. The bulky aldehyde was too large to be adsorbed within the channels, and this size selectivity thus demonstrated that the cooperatively catalytic $\beta$-arylation reaction mainly occurred in the channels of the polymer, not on the external surface (entry 7 , Table 1). To further test the impacts of external surface contact on the reaction, as-synthesized crystal samples (size: $50-80 \mu \mathrm{m}$ ) and finely ground particles (size: $0.1-1 \mu \mathrm{m}$ ) of InP-1 were used to catalyze the $\beta$-arylation reaction of 1,4-dicyanobenzene and benzenepropanal under the same reaction conditions (Supplementary Fig. 14). The resulting reactions gave the $\beta$-adduct with the comparable conversions of 52 and $56 \%$ after $24 \mathrm{~h}$ of irradiation, respectively, which demonstrated that the surface area was not the crucial factor for the transformation.

A systematic catalytic investigation was also interesting when comparing a previously reported polymer, MOF-150, involving $\mathbf{H}_{3}$ NTB as a building block ${ }^{44}$. As could be expected, a $5 \mathrm{~mol} \%$ loading of MOF-150 (per mole photosensitizer) under the same reaction conditions as the valeraldehyde system gave a negligible conversion. The excess addition of $5 \mathrm{~mol} \% \mathrm{~L}$-PYI to the reaction mixture led to a conversion of $52 \%$ but no ee value. Even with the addition increased to $20 \mathrm{~mol} \%$, only a $6 \%$ additional conversion and a negligible ee value could be detected (entries 2-4, Supplementary Table 7). The combination of $\mathbf{N T B}^{3-}$ and PYI groups in a single framework was not only advantageous to the 


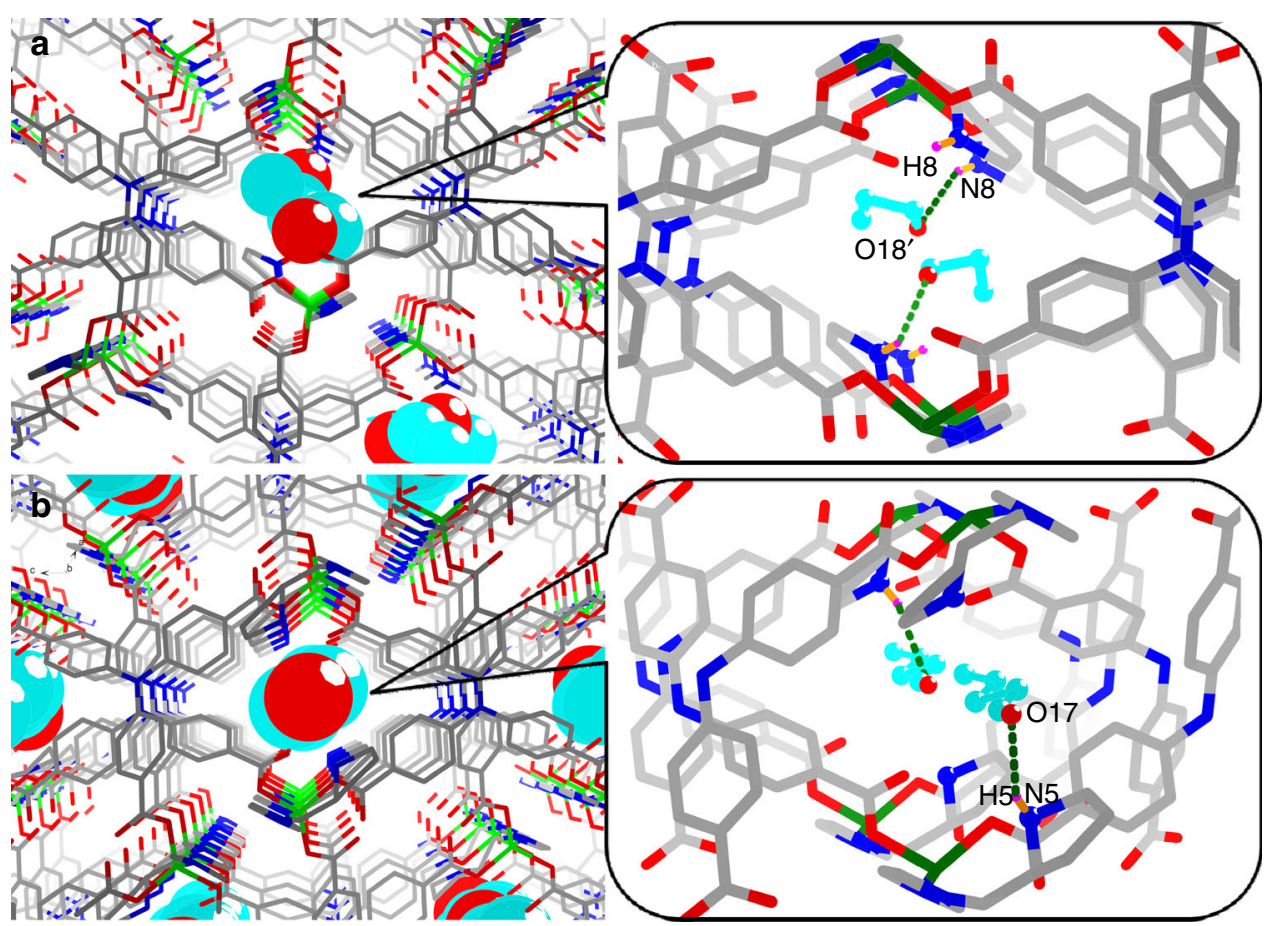

Fig. 5 Crystal structures of photocatalysts with absorbed substrates. Single-crystal structure of InP-4 (a) and InP-5 (b), showing the capture and activation of the substrates propionaldehyde and valeraldehyde, respectively, by hydrogen-bonding interactions in the channels of the crystals

efficient energy transfer and synergetic coupling of two catalytic cycles but also to the effective collision and constrained coupling between two activated radicals. The extension of the photocatalytic $\beta$-arylation of valeraldehyde to a previously reported non-interpenetrating $\mathrm{Zn}$-PYI $1^{25}$, which comprised both the $\mathrm{NTB}^{3-}$ and $L$-PYI groups, was also performed. With the shortest $\mathrm{N} \cdots \mathrm{N}$ separation of $10.9 \AA$ between the pyrrolidine and triphenylamine nitrogen atoms, the loading of an idential amount of $\mathrm{Zn}$-PYI1 under the same reaction conditions gave a conversion of $44 \%$ but negligible enantioselectivity. The modest catalytic activity and poor enantioselectivity for Zn-PYI1 may be caused by the longer distance between the catalytic centers in the photocatalyst and organocatalyst and the relatively uncontrolled pores generated by the stacked layers (Supplementary Fig. 13 and Supplementary Table 6). As the electron transfer from the enamine intermediate to the oxidized photoredox catalyst is the key step for the $\beta$-carbonyl activation, the superiority of an interpenetrated structure in heterogeneous systems is attributed to the close proximity between the two catalytic sites. This imposed vicinity encourages the direct interframework electron transfer for the $\beta$-activation of the carbonyl group to appear quickly and further promotes the formation of the $\beta$-enaminyl radicals via the critical $5 \pi \mathrm{e}^{-}$activation mode. The better enantioselectivity displayed by InP-1 is due to the wellmodified chirality-confined pores via framework interpenetration provided more matched and restricted chiral environments to force the substrate radicals to couple with each other in a specific direction and manner.

Photocatalytic $\beta$-functionalization of saturated ketones. The $5 \pi \mathrm{e}^{-}$carbonyl activation mode was also successfully applied in the photocatalytic $\beta$-coupling of cyclic ketenes with aryl ketones by MacMillan et al. under homogeneous conditions ${ }^{11}$. To further assess whether our MOF catalytic systems work for such reactions, InP-1 was employed to facilitate the direct $\beta$-functionalization of saturated ketones in a heterogeneous fashion. As shown in Fig. 6, the reaction was first evaluated using cyclohexanone and benzophenone as partners, along with $5 \mathrm{~mol} \%$ of InP-1, 2 equiv of DABCO and 1 equiv of $\mathrm{LiAsF}_{6}$ in DMF under visible-light irradiation. The resulting reaction gave the $\beta$-adduct with a conversion of $67 \%$ after $48 \mathrm{~h}$ without the formation of any $\alpha$ functionalization adduct, which revealed that the $5 \pi \mathrm{e}^{-} \beta$-ketone activation was indeed applied in such a heterogeneous system (Supplementary Table 8). Control experiments suggested that no desired product was detected in the absence of catalyst or light. When $5 \mathrm{~mol} \%$ of non-interpenetrating Zn-PYI1 was used for the same catalytic system, a very low conversion $(<5 \%)$ of the $\beta$ adduct and fairly complicated reaction products were detected. Such a result may be ascribed to the large separation of the catalytic sites and the more active ketone substrates; the unsatisfactory separation would greatly reduce the chance of the electron being transferred from the enamine intermediate to the oxidized photosensitizer and meanwhile would create new opportunities for other species. The superiority of the interpenetrated InP-1, with its closely spaced photoredox and organocatalytic sites, was extended to the $\beta$-coupling of cyclohexanone analogs (Supplementary Figs. 40-54), which led to conversions from 38 to $62 \%$ under the same reaction conditions (6b-e in Fig. 6). The availability of the $\beta$-adduct in such ketone systems benefited from the smooth initiation of the photoredox and organocatalytic cycles, which were confirmed by the emission quenching experiments of benzophenone and IR studies of the activation of ketones (Supplementary Figs. 26 and 27).

\section{Discussion}

We have reported a heterogeneous approach for the $\beta$-functionalization of saturated aldehydes and ketones under light irradiation and benign environments. The approach included the incorporation of photoredox and asymmetric catalytic groups in a single framework and the interpenetration of two enantiopure frameworks to modify electron transfer between the catalytic centers. The close proximity between the photoredox sites and the 

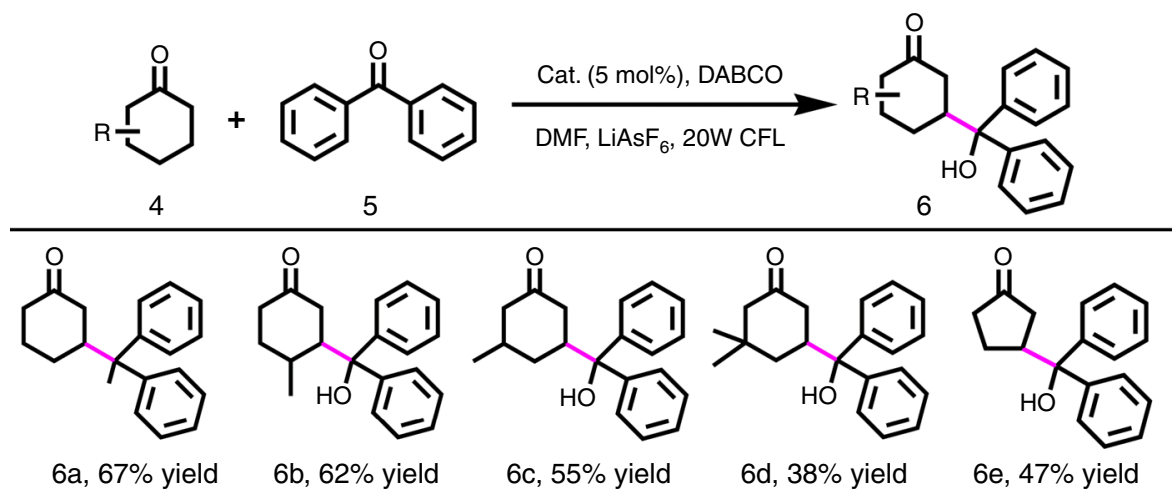

Fig. 6 Photocatalytic $\beta$-functionalization of ketones with InP-1 as catalyst. Reaction conditions: benzophenone ( $0.5 \mathrm{mmol})$, ketones (2.5 mmol), InP-1 ( $5 \mathrm{~mol} \%$, based on $\mathbf{H}_{3} \mathbf{N T B}$ ), LiAsF 6 ( $\left.0.5 \mathrm{mmol}\right), \mathrm{DABCO}(1.0 \mathrm{mmol})$, and DMF ( $\left.2 \mathrm{~mL}\right)$. The conversions were determined by GC-MS analysis

asymmetric organocatalytic sites via the framework interpenatration greatly increased the chance of enamine oxidation by the oxidized photosensitizer and the formation of the key $5 \pi \mathrm{e}^{-}$ enaminyl radicals. The modified environments of channels provided additional spatial constraints to enhance the regioselectivity and enantioselectivity of the $\beta$-activation products without introducing any additional chiral sources. The high selectivity, good recyclability, and clear interaction pattern render the interpenetration strategy highly promising for the regulation and optimization of reaction routes in heterogeneous photocatalysis, and allow the applied protocol to be used in the $\beta$-functionalization of saturated cyclic ketones with aryl ketones to deliver $\gamma$ hydroxyketone motifs.

\section{Methods}

Materials and measurements. Unless otherwise specified, all chemicals were of reagent-grade quality, were obtained from commercial sources and were used without further purification. $4,4^{\prime}, 4^{\prime \prime}$-Nitrilotrisbenzoicacid $\left(\mathbf{H}_{3} \mathbf{N T B}\right)^{45}, L$-/D-pyrrolidin-2-ylimidazole $(L-/ D \text {-PYI })^{46}$, MOF-150 ${ }^{44}$, and Zn-PYI ${ }^{25}$ were synthesized according to published procedures.

The elemental analyses of $\mathrm{C}, \mathrm{H}$, and $\mathrm{N}$ were performed on a Vario EL III elemental analyzer. FT-IR spectra were recorded from $\mathrm{KBr}$ pellets on a JASCO FT/ IR-430. The powder XRD diffractograms were obtained on a Rigaku D/Max-2400 $\mathrm{X}$-ray diffractometer with a sealed $\mathrm{Cu}$ tube $(\lambda=1.54178 \AA)$. Thermogravimetric analyses were performed at a ramp rate of $10^{\circ} \mathrm{C} / \mathrm{min}$ in a nitrogen flow with an SDTQ600 instrument. Sample morphologies were recorded via scanning electron microscopy on a Jeol JSM-6390A field emission scanning electron microscope.

${ }^{1} \mathrm{H}$ NMR spectra were recorded on a Varian INOVA-400M type spectrometer with chemical shifts reported as ppm (TMS as the internal standard). ${ }^{13} \mathrm{C} \mathrm{NMR}$ spectra were measured on a Varian INOVA-500M spectrometer with $\mathrm{CDCl}_{3}$ serving as an internal standard $(\delta=77.23)$. High resolution mass spectra were obtained on a GCT-CA156 Micromass GC/TOF mass spectrometer. GC analysis was performed on an Agilent Technologies 7820 A GC system. GC-MS studies were performed on a Thermo Scientific TRACE Ultra gas chromatographic instrument.

Solid UV-vis spectra were recorded on an HP 8453 spectrometer. Liquid UV-vis spectra were recorded on a TU-1900 spectrophotometer. Fluorescent spectra and lifetime measurements were recorded on an Edinburgh FS920 fluorescence spectrometer. All confocal laser scanning microscopy micrographs were collected by an Olympus Fluoview FV1000 with $\lambda_{\mathrm{ex}}=488 \mathrm{~nm}$.

HPLC analysis was performed on an Agilent 1100 using a ChIRALPAK AS-H column purchased from Daicel Chemical Industries, Ltd. Products were purified by flash column chromatography on 200-300 mesh silica gel and Kromasil Lc-80 using a $\mathrm{SiO}_{2}$ chromatographic column. The $\mathrm{CD}$ spectra were measured on a JASCO J-810 by grinding the crystalline sample into powder to be recorded as $\mathrm{KBr}$ pellets.

Solid-state cyclic voltammograms were recorded on a Zahner PP211 instrument. The detailed experiments were performed with a commonly used three-electrode system (working electrode-an improved glassy carbon electrode; reference electrode- $\mathrm{Ag} / \mathrm{AgCl}$ electrode; auxiliary electrode-platinum wire) in phosphate-buffered saline (PBS; scan rate: $50 \mathrm{mV} \mathrm{s}^{-1}$; scan range: $0.4-1.2 \mathrm{~V}$ ).

X-ray crystallography. Single-crystal XRD data were collected on a Bruker SMART APEX diffractometer equipped with a CCD area detector and a Mo-K $\alpha$ $(\lambda=0.71073 \AA)$ radiation source. The data integration and reduction were processed using SAINT software ${ }^{47}$. An empirical absorption correction was applied to the collected reflections with SADABS ${ }^{48}$. The structures were solved by direct methods using SHELXTL and were refined on $F^{2}$ by the full-matrix least-squares method using the SHELXL-97 program ${ }^{49,50}$. All non-hydrogen atoms in the backbone of the polymers were refined anisotropically until convergence was reached. Hydrogen atoms attached to the organic ligands were located geometrically and refined in a riding model, whereas some of the disordered solvent molecules were not treated during the structural refinements. To assist in the stability of the refinement, bond distances between several disordered atoms were fixed. Crystallographic data for InP-1, InP-2, InP-3, InP-4 and InP-5 are summarized in Supplementary Table 2.

Syntheses of InP-1 and InP-2. A mixture of $\mathbf{H}_{3}$ NTB $(37.7 \mathrm{mg}, 0.1 \mathrm{mmol}), \mathrm{Zn}$ $\left(\mathrm{NO}_{3}\right)_{2} \cdot 6 \mathrm{H}_{2} \mathrm{O}(29.8 \mathrm{mg}, 0.1 \mathrm{mmol})$ and $L$-PYI $(15.1 \mathrm{mg}, 0.1 \mathrm{mmol})$ for InP-1 or $D$ PYI (15.1 mg, $0.1 \mathrm{mmol})$ for InP-2 was dissolved in mixed water $(3.0 \mathrm{~mL}), \mathrm{CH}_{3} \mathrm{OH}$ $(1.0 \mathrm{~mL})$, and DMF $(2.0 \mathrm{~mL})$. The resulting solution was stirred for $\sim 30 \mathrm{~min}$ at room temperature, sealed in a $10 \mathrm{~mL}$ Teflon-lined stainless steel autoclave, and heated at $110^{\circ} \mathrm{C}$ for 3 days under autogenous pressure. The reaction system was then cooled to room temperature at a rate of $5^{\circ} \mathrm{Ch}^{-1}$. Yellow block crystals were collected in $47 \%$ yield (based on $\mathrm{Zn}$ ). Elemental analysis (\%) Calc. for $\mathrm{C}_{120} \mathrm{H}_{125} \mathrm{~N}_{17} \mathrm{O}_{31} \mathrm{Zn}_{4}: \mathrm{H}, 4.92 ; \mathrm{C}, 56.24 ; \mathrm{N}, 9.29$. Found: $\mathrm{H}, 5.12 ; \mathrm{C}, 56.78 ; \mathrm{N}, 8.88$ for InP-1; Found: H, 4.98; C, 56.13; N, 8.97 for InP-2.

Synthesis of InP-3. A mixture of $\mathbf{H}_{3} \mathrm{NTB}$ (37.7 mg, $0.1 \mathrm{mmol}$ ), $\mathrm{Zn}\left(\mathrm{NO}_{3}\right)_{2} \cdot 6 \mathrm{H}_{2} \mathrm{O}$ $(29.8 \mathrm{mg}, 0.1 \mathrm{mmol})$ was dissolved in mixed water $(1.0 \mathrm{~mL})$, pyridine $(1.0 \mathrm{~mL})$, and DMF $(4.0 \mathrm{~mL})$. The resulting solution was stirred for $\sim 30 \mathrm{~min}$ at room temperature, sealed in a $10 \mathrm{~mL}$ Teflon-lined stainless steel autoclave, and heated at $110^{\circ} \mathrm{C}$ for 3 days under autogenous pressure. The reaction system was then cooled to room temperature at a rate of $5{ }^{\circ} \mathrm{Ch}^{-1}$. Light-yellow block crystals were collected in $56 \%$ yield (based on $\mathrm{Zn}$ ). Elemental analysis (\%) Calc. for $\mathrm{C}_{115} \mathrm{H}_{115} \mathrm{~N}_{13} \mathrm{O}_{29} \mathrm{Zn}_{4}: \mathrm{H}$, 4.82; C, 57.44.; N, 7.57. Found: H, 4.72; C, 57.89; N, 7.66.

Synthesis of InP-4. Crystals of InP-4 were obtained by soaking the crystals of InP-1 $(0.01 \mathrm{mmol})$ in a DMF $(1.5 \mathrm{~mL})$ solution containing propionaldehyde $(0.5$ mmol) for $12 \mathrm{~h}$. Elemental analysis (\%) Calc. for $\mathrm{C}_{125} \mathrm{H}_{134} \mathrm{~N}_{16} \mathrm{O}_{33} \mathrm{Zn}_{4}: \mathrm{H}, 5.10$; C, 56.65.; N, 8.46. Found: H, 4.93; C, 56.92; N, 8.36 .

Synthesis of InP-5. Crystals of InP-5 were obtained by soaking the crystals of InP$2(0.01 \mathrm{mmol})$ in a DMF $(1.5 \mathrm{~mL})$ solution containing valeraldehyde $(0.5 \mathrm{mmol})$ for 12 h. Elemental analysis (\%) Calc. for $\mathrm{C}_{128} \mathrm{H}_{140} \mathrm{~N}_{16} \mathrm{O}_{32} \mathrm{Zn}_{4}: \mathrm{H}, 5.27$;, 57.45 .; $\mathrm{N}$, 8.37. Found: $\mathrm{H}, 4.98 ; \mathrm{C}, 57.12 ; \mathrm{N}, 8.56$

General procedure for the $\boldsymbol{\beta}$-functionalization of saturated aldehydes. A glass tube was filled with 1,4 -dicyanobenzene $(1.0 \mathrm{mmol})$, catalyst $(0.025 \mathrm{mmol})$, and 1,4-diazabicyclo[2.2.2] octane (DABCO, $5.0 \mathrm{mmol}$ ). Then, this vial was purged with $\mathrm{N}_{2}$, and the corresponding aldehyde $(1.4 \mathrm{mmol})$ and DMF $(3.0 \mathrm{~mL})$ were added via syringe. The resulting mixture was then cooled to $-78^{\circ} \mathrm{C}$, degassed and backfilled with $\mathrm{N}_{2}$ three times. The vial was placed $\sim 5 \mathrm{~cm}$ away from a $20 \mathrm{~W}$ fluorescent lamp source for $48 \mathrm{~h}$ of irradiation. The reaction mixture was extracted with ethyl acetate, dried with anhydrous $\mathrm{Na}_{2} \mathrm{SO}_{4}$, concentrated in vacuo and purified by flash chromatography on silica gel using hexane/ethyl acetate as the eluent to give the $\beta$ arylated aldehyde product as an oil. For the aromatic aldehyde substrates, the supernatant solution after centrifugation was diluted with $8 \mathrm{~mL}$ of DCM and $2 \mathrm{~mL}$ of $\mathrm{MeOH}$. The reaction mixture was then cooled to $0{ }^{\circ} \mathrm{C}$ and sodium borohydride ( $5.0 \mathrm{mmol}, 5.0$ equiv) was added to reduce the resulting aldehyde to the 
corresponding alcohol. Then the reaction mixture was treated with an identical procedure for the aliphatic aldehyde product.

General procedure for the $\boldsymbol{\beta}$-functionalization of saturated ketones. A glass tube was filled with benzophenone $(0.5 \mathrm{mmol})$, catalyst $(12.5 \mu \mathrm{mol}), \operatorname{LiAsF}_{6}(0.5$ $\mathrm{mmol})$, and DABCO $(1.0 \mathrm{mmol})$. Then, this vial was purged with $\mathrm{N}_{2}$, and the corresponding cyclohexanone $(2.5 \mathrm{mmol}, 5.0$ equiv), and DMF $(2.0 \mathrm{~mL})$ was added via syringe. The resulting mixture was then cooled to $-78^{\circ} \mathrm{C}$, degassed and backfilled with $\mathrm{N}_{2}$ three times. The vial was placed $\sim 5 \mathrm{~cm}$ away from a $20 \mathrm{~W}$ fluorescent lamp source for $48 \mathrm{~h}$ of irradiation. The reaction mixture was diluted with water and extracted with ethyl acetate. The combined organic layers were washed with brine, dried with anhydrous $\mathrm{Na}_{2} \mathrm{SO}_{4}$, concentrated in vacuo, and purified by flash chromatography on silica gel using hexane/ethyl acetate as the eluent to provide an inseparable mixture of the desired $\beta$-adduct and the corresponding hemiacetal.

General procedure for dye-uptake measurements. Before the adsorption of dye, InP-1 was first soaked in methanol solution $(24 \mathrm{~h})$ for guest molecule exchange and then fully dried in a vacuum oven $\left(120^{\circ} \mathrm{C}, 12 \mathrm{~h}\right)$ to eliminate the guest molecules (Supplementary Fig. 3). Then, the dried InP-1 $(2.56 \mathrm{mg}, 2 \mu \mathrm{mol})$ was soaked in a methanol solution of $2^{\prime}, 7^{\prime}$-dichlorofluorescein dye $(24 \mathrm{mM}, 2 \mathrm{~mL})$ in a constant temperature oscillation incubator overnight. The resulting InP-1 was filtered and washed with methanol thoroughly until the solution became colorless and was then dried under a stream of air. The dried samples were dissociated by concentrated hydrochloric acid, and the resultant clear solution with a light olivine color was diluted to $10 \mathrm{~mL}$ and adjusted to a $\mathrm{pH}$ of 1.5 . The dye concentration was determined by comparing the solution UV-vis absorption with a standard curve of the dye.

Data availability. The X-ray crystallographic coordinates for structures reported in this Article have been deposited at the Cambridge Crystallographic Data Centre (CCDC) under deposition numbers CCDC 1510628-1510630 and 15402311540232 (Supplementary Table 2). These data can be obtained free of charge from The Cambridge Crystallographic Data Centre via www.ccdc.cam.ac.uk/ data_request/cif. All other data supporting the findings of this study are available within the Article and its Supplementary Information files, or from the corresponding author on reasonable request.

Received: 3 January 2017 Accepted: 26 June 2017

Published online: 25 August 2017

\section{References}

1. Yoon, T. P., Ischay, M. A. \& Du, J. Visible light photocatalysis as a greener approach to photochemical synthesis. Nat. Chem. 2, 527-532 (2010).

2. Evans, D. A., Helmchen, G. \& Rüping, M. Asymmetric Synthesis-The Essentials (Wiley-VCH, 2006).

3. Mukherjee, S., Yang, J. W., Hoffmann, S. \& List, B. Asymmetric enamine catalysis. Chem. Rev. 107, 5471-5569 (2007).

4. Carey, F. A. \& Sundberg, R. J. Advanced Organic Chemistry: Part B: Reactions and Synthesis (Springer, 2001).

5. Trost, B. M. \& Fleming, I. Comprehensive Organic Synthesis, Vol. 2 (Pergamon, 1991).

6. Johansson, C. C. C. \& Colacot, T. J. Metal-catalyzed $\alpha$-arylation of carbonyl and related molecules: novel trends in $\mathrm{C}-\mathrm{C}$ bond formation by $\mathrm{C}-\mathrm{H}$ bond functionalization. Angew. Chem. Int. Ed. 49, 676-707 (2010).

7. Melchiorre, P. Cinchona-based primary amine catalysis in the asymmetric functionalization of carbonyl compounds. Angew. Chem. Int. Ed. 51, 9748-9770 (2012).

8. Nicewicz, D. A. \& MacMillan, D. W. C. Merging photoredox catalysis with organocatalysis: the direct asymmetric alkylation of aldehydes. Science 322, 77-80 (2008).

9. Zhang, S. L. et al. Organocatalytic enantioselective $\beta$-functionalization of aldehydes by oxidation of enamines and their application in cascade reactions. Nat. Commun. 2, 211-218 (2011).

10. Huang, Z. \& Dong, G. Catalytic direct $\beta$-arylation of simple ketones with aryl iodides. J. Am. Chem. Soc. 135, 17747-17750 (2013).

11. Petronijević, F. R., Nappi, M. \& MacMillan, D. W. C. Direct $\beta$-functionalization of cyclic ketones with aryl ketones via the merger of photoredox and organocatalysis. J. Am. Chem. Soc. 135, 18323-18326 (2013).

12. Ibrahem, I., Ma, G., Afewerki, S. \& Córdova, A. Palladium/chiral amine cocatalyzed enantioselective $\beta$-arylation of $\alpha, \beta$-unsaturated aldehydes. Angew. Chem. Int. Ed. 52, 878-882 (2013).

13. Itooka, R., Iguchi, Y. \& Miyaura, N. Rhodium-catalyzed 1,4-addition of arylboronic acids to $\alpha, \beta$-unsaturated carbonyl compounds: large accelerating effects of bases and ligands. J. Org. Chem. 68, 6000-6004 (2003).
14. Leskinen, M. V., Yip, K.-T., Valkonen, A. \& Pihko, P. M. Palladium-catalyzed dehydrogenative $\beta^{\prime}$-functionalization of $\beta$-keto esters with indoles at room temperature. J. Am. Chem. Soc. 134, 5750-5753 (2012).

15. Wasa, M., Engle, K. M. \& Yu, J.-Q. $\mathrm{Pd}(0) / \mathrm{PR}_{3}$-Catalyzed intermolecular arylation of $\mathrm{sp}^{3}$ C-H bonds. J. Am. Chem. Soc. 131, 9886-9887 (2009).

16. Prier, C. K., Rankic, D. A. \& MacMillan, D. W. C. Visible light photoredox catalysis with transition metal complexes: applications in organic synthesis. Chem. Rev. 113, 5322-5363 (2013).

17. Schultz, D. M. \& Yoon, T. P. Solar synthesis: prospects in visible light photocatalysis. Science 343, 1239176-1-1239176-8 (2014).

18. Pirnot, M. T., Rankic, D. A., Martin, D. B. C. \& MacMillan, D. W. C. Photoredox activation for the direct $\beta$-arylation of ketones and aldehydes. Science 339, 1593-1596 (2013).

19. Lee, J. et al. Metal-organic framework materials as catalysts. Chem. Soc. Rev. 38 1450-1459 (2009).

20. Yoon, M., Srirambalaji, R. \& Kim, K. Homochiral metal-organic frameworks for asymmetric heterogeneous catalysis. Chem. Rev. 112, 1196-1231 (2012).

21. Shi, D. et al. Merging of the photocatalysis and copper catalysis in metal-organic frameworks for oxidative C-C bond formation. Chem. Sci. 6, 1035-1042 (2015).

22. Zeng, L. et al. Organized aggregation makes insoluble perylene diimide efficient for the reduction of aryl halides via consecutive visible light-induced electrontransfer processes. J. Am. Chem. Soc. 138, 3958-3961 (2016).

23. Zhang, Q. et al. Förster energy transport in metal-organic frameworks is beyond step-by-step hopping. J. Am. Chem. Soc. 138, 5308-5315 (2016).

24. Son, H.-J. et al. Light-harvesting and ultrafast energy migration in porphyrinbased metal-organic frameworks. J. Am. Chem. Soc. 135, 862-869 (2013).

25. Wu, P. et al. Photoactive chiral metal-organic frameworks for light-driven asymmetric $\alpha$-alkylation of aldehydes. J. Am. Chem. Soc. 134, 14991-14999 (2012).

26. Dias, L. C., Marchi, A. A., Ferreira, M. A. B. \& Aguilar, A. M. 1,5-Asymmetric induction in boron-mediated aldol reactions of $\beta$-alkoxy methylketones. J. Org. Chem. 73, 6299-6311 (2008).

27. Spek, A. L. PLATON99, A Multipurpose Crystallographic Tool (Utrecht University, 1999)

28. Fang, Q. R. et al. Mesoporous metal-organic framework with rare etb topology for hydrogen storage and dye assembly. Angew. Chem., Int. Ed. 46, 6638-6642 (2007).

29. Ma, L., Falkowski, J. M., Abney, C. \& Lin, W. A series of isoreticular chiral metal-organic frameworks as a tunable platform for asymmetric catalysis. Nat. Chem. 2, 838-846 (2010).

30. Zhang, L. et al. Post-modification of a MOF through a fluorescent-labeling technology for the selective sensing and adsorption of $\mathrm{Ag}^{+}$in aqueous solution. Dalton Trans. 41, 10153-10155 (2012).

31. Shin, S. M., Lee, M. S., Han, J. H. \& Jeong, N. Assessing the guest-accessible volume in MOFs using two-photon fluorescence microscopy. Chem. Commun. 50, 289-291 (2014).

32. Horike, S., Dincă, M., Tamaki, K. \& Long, J. R. Size-selective lewis acid catalysis in a microporous metal-organic framework with exposed $\mathrm{Mn}^{2+}$ coordination sites. J. Am. Chem. Soc. 130, 5854-5855 (2008).

33. Adelizzi, B., Filot, I. A. W., Palmans, A. R. A. \& Meijer, E. W. Unravelling the pathway complexity in conformationally flexible $\mathrm{N}$-centered triarylamine trisamides. Chem. Eur. J. 22, 6103-6110 (2017).

34. Deng, S., Chen, T., Chien, W. \& Hong, J. Aggregation-enhanced emission in fluorophores containing pyridine and triphenylamine terminals: restricted molecular rotation and hydrogen-bond interaction. J. Mater. Chem. C 2, 651-659 (2014).

35. Meijer, G., Berden, G. \& Meerts, W. L. Spectroscopy on triphenylamine and its van der Waals complexes. Chem. Phys. 163, 209-222 (1992).

36. Kavarnos, G. J. Fundamentals of Photoinduced Electron Transfer (VCH, 1993)

37. Dixon, I. M. et al. A family of luminescent coordination compounds: iridium (III) polyimine complexes. Chem. Soc. Rev. 29, 385-391 (2000).

38. Flamigni, L., Barbieri, A., Sabatini, C., Ventura, B. \& Barigelletti, F. Photochemistry and photophysics of coordination compounds: iridium. Top. Curr. Chem. 281, 143-203 (2007).

39. Mori, Y., Sakaguchi, Y. \& Hayashi, H. Magnetic field effects on chemical reactions of biradical radical ion pairs in homogeneous fluid solvents. J. Phys. Chem. A 104, 4896-4905 (2000).

40. Jing, X., He, C., Yang, Y. \& Duan, C. A metal-organic tetrahedron as a redox vehicle to encapsulate organic dyes for photocatalytic proton reduction. J. Am. Chem. Soc. 137, 3967-3974 (2015).

41. Inokuma, Y. et al. X-ray analysis on the nanogram to microgram scale using porous complexes. Nature 495, 461-466 (2013).

42. Lee, S., Kapustin, E. A. \& Yaghi, O. M. Coordinative alignment of molecules in chiral metal-organic frameworks. Science 353, 808-811 (2016).

43. Banerjee, M. et al. Postsynthetic modification switches an achiral framework to catalytically active homochiral metal-organic porous materials. J. Am. Chem. Soc. 131, 7524-7525 (2009).

44. Chae, H. K., Kim, J., Friedrichs, O. D., O’Keeffe, M. \& Yaghi, O. M. Design of frameworks with mixed triangular and octahedral building blocks exemplified by the structure of $\left[\mathrm{Zn}_{4} \mathrm{O}(\mathrm{TCA})_{2}\right]$ having the pyrite topology. Angew. Chem. Int. Ed. 42, 3907-3909 (2003). 
45. Wang, J., He, C., Wu, P., Wang, J. \& Duan, C. An amide-containing metal-organic tetrahedron responding to a spin-trapping reaction in a fluorescent enhancement manner for biological imaging of NO in living cells. J. Am. Chem. Soc. 133, 12402-12405 (2011).

46. Dang, D., Wu, P., He, C., Xie, Z. \& Duan, C. Homochiral metal-organic frameworks for heterogeneous asymmetric catalysis. J. Am. Chem. Soc. 132, 14321-14323 (2010).

47. SAINT, Data reduction software (version 6.45) (Bruker AXS Inc., Madison, WI, 2003).

48. Sheldrick, G. M. SADABS Empirical Absorption Program (University of Göttingen, 1996).

49. Sheldrick, G. M. SHELXTL Reference Manual: Version 5.1 (Bruker AXS, 1997).

50. Sheldrick, G. M. SHELXL-97: Program for Crystal Structure Refinement (University of Göttingen, 1997).

\section{Acknowledgements}

This study was supported by the National Natural Science Foundation of China (U1608224, 21421005, and 21231003).

\section{Author contributions}

Z.X., C.H. and C.D. conceived and designed the project. Z.X. and X.W. performed the experiments. C.H. and C.D. contributed materials and analysis tools. Z.X., C.H. and C.D. co-wrote the paper. All authors discussed the results and commented on the manuscript.

\section{Additional information}

Supplementary Information accompanies this paper at doi:10.1038/s41467-017-00416-8.

Competing interests: The authors declare no competing financial interests.

Reprints and permission information is available online at http://npg.nature.com/ reprintsandpermissions/

Publisher's note: Springer Nature remains neutral with regard to jurisdictional claims in published maps and institutional affiliations.

cc) (i) Open Access This article is licensed under a Creative Commons Attribution 4.0 International License, which permits use, sharing, adaptation, distribution and reproduction in any medium or format, as long as you give appropriate credit to the original author(s) and the source, provide a link to the Creative Commons license, and indicate if changes were made. The images or other third party material in this article are included in the article's Creative Commons license, unless indicated otherwise in a credit line to the material. If material is not included in the article's Creative Commons license and your intended use is not permitted by statutory regulation or exceeds the permitted use, you will need to obtain permission directly from the copyright holder. To view a copy of this license, visit http://creativecommons.org/ licenses/by/4.0/.

(C) The Author(s) 2017 